\title{
A MULTILEVEL METHOD OF NONLINEAR GALERKIN TYPE FOR THE NAVIER-STOKES EQUATIONS
}

\author{
SAID EL HAJJI AND KHALID ILIAS
}

Received 21 October 2004

The basic idea of this new method resides in the fact that the major part of the relative information to the solution to calculate is contained in the small modes of a development of Fourier series; the raised modes of which the coefficients associated being small, being negligible to every instant, however, the effect of these modes on a long interval of time is not negligible. The nonlinear Galerkin method proposes economical treatment of these modes that permits, in spite of a simplified calculation, taking into account their interaction correctly with the other modes. After the introduction of this method, we elaborate an efficient strategy for its implementation.

\section{Introduction}

The numerical integration of the Navier-Stokes equations on large intervals of time yields new problems and new challenges with which we will be faced in the coming years. Indeed, the considerable increase in the computing power during the last years makes it thinkable to solve these equations and similar ones in dynamically nontrivial situations.

In relation with the recent developments in the theory of dynamical systems and its application to the theoretical survey of the turbulent phenomena (attractors, inertial manifolds), new algorithms have been introduced by Foias et al. in [6], as well as Marion and Temam in [12].

These methods of multiresolution, also named nonlinear Galerkin methods, essentially apply to the approximation of nonlinear dissipative systems, as the equations of Navier-Stokes. Based on a decomposition of the unknowns, as the velocity field, into small and large eddies, Foias, Manley, and Temam defined new objects: the approximate inertial manifolds [6]. These manifolds define an adiabatic law, modeling the interaction of the different structures of the flow, the small structures are in fact expressed as a nonlinear function of large scales. Moreover, these Manifolds enjoy the property that they attract all the orbits exponentially fast in time and that they contain the attractor in a thin neighborhood. They provide a good way to approach the solutions of the Navier-Stokes equations. 
These approximate inertial manifolds are subsets of the phase space and consist of an approximation form of the small scale equations.

The nonlinear Galerkin method, proposed by Marion and Temam [12], consists of looking for a solution lying on these specific subsets of the phase space.

The first computational tests of this new method were conducted by Jauberteau [10], Jauberteau et al. [11] in the bidimensional case, where the exact solutions of the equations were known, they seem appropriate for long time integration of Navier-Stokes equations.

Numerical simulation of turbulent flows being performed at a small fraction of the computational effort is usually required by traditional methods, see [4].

Our aim in this article is to study the implementation of the nonlinear Galerkin method in the context of pseudospectral discretization for the three-dimensional NavierStokes equations. Other aspects of multilevel methods of Galerkin type appear in [5] by Dubois et al.

After describing the method, we report on numerical computations based on this approach. They show an improvement in stability and precision and a significant gain in computing time.

The calculations of Examples 1 and 2 have been, respectively, carried out on the Cray-2 and Titan.

\section{The nonlinear Galerkin method}

In this part, we consider the incompressible flows of which the velocity field $u=\left(u_{1}, u_{2}\right.$, $u_{3}$ ) in dimension 3 verifies the Navier-Stokes equations:

$$
\begin{gathered}
\frac{\partial u}{\partial t}-v \Delta u+(w \times u)+\frac{1}{2} \nabla|u|^{2}+\nabla p=f, \\
\nabla \cdot u=0, \\
u(x, t=0)=u_{0}(x),
\end{gathered}
$$

where $v$ is the kinematic viscosity, $w(x, t)=\nabla \times u(x, t)$ the vorticity, $p$ the pressure, and $f$ the external force.

Here, $|-|$ stands for the Euclidean norm in $\mathbb{R}^{3}$.

Moreover, we impose $u$ and $p$ to be periodic in space. Hence, they can be expanded in Fourier series, namely,

$$
u(x, t)=\sum_{k \in \mathbb{Z}^{3}} \widehat{u}_{k}(t) e^{i k \cdot x}
$$

and similarly for $f(x, t)$ and $p(x, t)$.

We now introduce the orthogonal projection $P_{\text {div }}$ onto the divergence free space; $P_{\text {div }}$ can be easily expressed as

$$
P_{\operatorname{div}} \phi(x)=\sum_{k \in \mathbb{Z}^{3}}\left(\hat{\phi}_{k}-\frac{k}{|k|^{2}}\left(k \cdot \hat{\phi}_{k}\right)\right) e^{i k \cdot x},
$$

where $\phi(x)=\sum_{k \in \mathbb{Z}^{3}} \hat{\phi}_{k} e^{i k \cdot x}$. 
Assuming that $u$ and $p$ lie in the proper Hilbert spaces and applying $P_{\text {div }}$ to the NavierStokes equations (2.1) can be put then under the following abstract form:

$$
\frac{\partial u}{\partial t}-v \triangle u+B(u, u)=g
$$

where $g=P_{\operatorname{div}} f$ and $B(u, u)$ is a bilinear form defined by

$$
\begin{aligned}
B(u, u) & =P_{\operatorname{div}}(w \times u) \\
& \left.=\sum_{k \in \mathbb{Z}^{3}}(\widehat{(w \times u)})_{k}-\frac{k}{|k|^{2}} k \cdot(\widehat{w \times u})_{k}\right) e^{i k \cdot x} .
\end{aligned}
$$

The numerical procedures are directly applied to this last form of the Navier-Stokes equations. This formulation is very useful in practice and allows to reduce the memory size of the codes.

Based on the limit conditions, it is natural to approach (2.6) by a pseudospectral Galerkin method [2], based on a development of $u$ in Fourier series.

We introduce the following decomposition:

$$
u_{N}=y_{N_{1}}+z_{N_{1}} \quad \text { with } N_{1} \leq N
$$

where

$$
\begin{aligned}
& y_{N_{1}}=P_{N_{1}} u_{N}, \\
& z_{N_{1}}=Q_{N_{1}} u_{N} .
\end{aligned}
$$

$P_{N_{1}}$ and $Q_{N_{1}}$ are operators of projection onto the space of Fourier. $y_{N 1}$ represents the large scales (structures) of the flow, $z_{N_{1}}$ the small scales.

After projection of (2.6) on the spaces $P_{N_{1}}$ and $Q_{N_{1}}$, the variables $y_{N_{1}}$ and $z_{N_{1}}$ are then solution of the coupled system according to

$$
\begin{aligned}
& \frac{d y_{N_{1}}}{d t}-v \triangle y_{N_{1}}+P_{N_{1}} B\left(y_{N_{1}}+z_{N_{1}}, y_{N_{1}}+z_{N_{1}}\right)=P_{N_{1}} g, \\
& \frac{d z_{N_{1}}}{d t}-v \triangle z_{N_{1}}+Q_{N_{1}} B\left(y_{N_{1}}+z_{N_{1}}, y_{N_{1}}+z_{N_{1}}\right)=Q_{N_{1}} g .
\end{aligned}
$$

Due to the bilinearity of $B$, we can split the nonlinear term $B\left(u_{N}, u_{N}\right)$ into

$$
\begin{aligned}
B\left(u_{N}, u_{N}\right) & =B\left(y_{N_{1}}+z_{N_{1}}, y_{N_{1}}+z_{N_{1}}\right) \\
& =B\left(y_{N_{1}}, y_{N_{1}}\right)+B_{\text {int }}\left(y_{N_{1}}, z_{N_{1}}\right),
\end{aligned}
$$

where $B\left(y_{N_{1}}, y_{N_{1}}\right)$ is the nonlinear term associated to $y_{N_{1}}$, and $B_{\text {int }}\left(y_{N_{1}}, z_{N_{1}}\right)=B\left(y_{N_{1}}, z_{N_{1}}\right)$ $+B\left(z_{N_{1}}, y_{N_{1}}\right)+B\left(z_{N_{1}}, z_{N_{1}}\right)$ is the coupling term and interaction between small and large structures.

In [6], Foias et al. showed that for $N$ and $N_{1}$ sufficiently large and after one period of transition depending on the data, some quantities functions of $z$ are negligible in relation 
to the other terms of the equation. On the other hand, since the evolution of $z$, compared to $y$, is quasistatic, we will study the approached system according to

$$
\begin{gathered}
\frac{d y_{N_{1}}}{d t}-v \triangle y_{N_{1}}+P_{N_{1}} B\left(y_{N_{1}}, y_{N_{1}}\right)+P_{N_{1}} B_{\text {int }}\left(y_{N_{1}}, z_{N_{1}}\right)=P_{N_{1}} g \\
-v \triangle z_{N_{1}}+Q_{N_{1}} B\left(y_{N_{1}}, y_{N_{1}}\right)=Q_{N_{1}} g .
\end{gathered}
$$

The nonlinear Galerkin method introduced by Marion and Temam [12] and Foias et al. [6] consists of looking for an approximation of the solution $u$ given by

$$
u_{N}=y_{N_{1}}+z_{N_{1}} \quad \text { with } N_{1} \leq N
$$

and $u_{N}$ satisfied the system (2.12), (2.13).

We recall that the classical (usual) Galerkin method of $u$ consists of putting $z_{N_{1}}=0$ in (2.12), therefore $P_{N_{1}} B_{\text {int }}\left(y_{N_{1}}, z_{N_{1}}\right)$ is neglected.

Equation (2.13) provides a nonlinear interaction law between large and small structures. It is the equation of approximate inertial manifold [6].

The small scales are explicitly (approximately) given in terms of the large scales by

$$
z_{N_{1}}=\phi\left(y_{N_{1}}\right)=(\nu \triangle)^{-1}\left(Q_{N_{1}} B\left(y_{N_{1}}, y_{N_{1}}\right)-Q_{N_{1}} g\right)
$$

\section{Description of the multilevel method}

The small scales and the coupling terms can be fixed in time during few iterations. However, the order of their size can change rigorously during one period of time. So the cutoff $N_{1}$ defining the separation of the small and large scales cannot be fixed in time. We propose a multilevel adaptative procedure valuing the appropriate levels of the refinement in time, by using the theoretical arguments in [6]. The implementation is achieved then by a succession of cycles defined by two levels of the cutoff $N_{i_{1}}$ and $N_{i_{2}}$ as in the classical multigrid methods $[1,8]$.

One chooses a number $N$ that represents the total number of the modes retained of the solution $u$ by truncation

$$
u_{N}=\sum_{k \in I_{N}} \hat{u}_{k}(t) e^{i k \cdot x}
$$

where $I_{N}=[1-N / 2, N / 2]^{3}$.

$N$ is valued according to the following criteria:

(i) convergence of the truncated series $u_{N}$ : the energy spectrum having a zone of strong decrease for $k$ large (viscous zone in turbulence);

(ii) for nonexact solutions, we have to estimate the number of degrees of liberties required for a correct description of the attractor (evaluation via the number of Reynolds).

Once $N$ is determined, we choose a time step $\triangle t$ taking account of the restriction coming from the numerical stability. 
Knowing the maximal number $N$ of modes, we define values $N_{i}$ determining coarse grids

$$
N_{1}<N_{2}<\cdots<N_{i}<N_{i+1}<\cdots<N
$$

which are going to allow us to decompose the solution $u_{N}$ under the form

$$
u_{N}=y_{N_{i}}+z_{N_{i}}
$$

One must have $N_{i}$ under the form $2^{p} \times 3^{q} \times 5^{r}, p \geq 2, q, r \in \mathbb{N}$, to permit the use of the fast Fourier transform (FFT).

The scheme of temporal integration for the nonlinear Galerkin method is based on a multigrid cycle and a quasistatic integration of the most high modes of the approximation $u_{N}$.

In the beginning of the cycle, we suppose that the approximation $u_{N}$ is known at the time $t_{n-1}=(n-1) \Delta t$. The computation of $u_{N}$ at the instant $t_{n}$ is done by the integration of the system (2.6) as for the pseudospectral Galerkin method, that is, without separation of the scales, by a method of quadrature for the linear term and an explicit Runge-Kutta method of order 3 for the nonlinear term.

Knowing $u_{N}$ at the instant $t_{n}$, we define two coarse levels $N_{i_{1}}\left(t_{n}\right)$ and $N_{i_{2}}\left(t_{n}\right)$ by the following tests: $i_{1}$ is determined by the condition

$$
\frac{\left|z_{N_{i}}\left(t_{n}\right)\right|_{2}}{\left|y_{N_{i}}\left(t_{n}\right)\right|_{2}}<\operatorname{Tol} 0 \quad \forall i \geq i_{1} \text {, }
$$

$i_{2}$ is determined by the condition

$$
\frac{\left|z_{N_{i}}\left(t_{n}\right)\right|_{2}}{\left|y_{N_{i}}\left(t_{n}\right)\right|_{2}}<\text { Tol1 } \quad \forall i \geq i_{2},
$$

where Tol 0 and Tol 1 are two constants given and fixed in the beginning of the cycle. $|\cdot|_{2}$ is the norm in $L^{2}$.

The test (3.5) assures us that $\left|z_{N_{i_{2}}}\right|_{2}$ is the order of the precision of the numerical scheme of integration in time $\left(\triangle t^{3}\right)$.

The test (3.4) assures us a predominance of $y_{N_{i}}$ before $z_{N_{i}}$ for all $i \geq i_{1} . N_{i_{1}}$ defines the minimal level also on which we can use the quasistatic approximation again for the high modes $z$ and justify the fact to choose the nonlinear terms of interaction with their value to the last instant of integration of $z$. The refinement of the levels between $N_{i_{1}}\left(t_{n}\right)$ and $N_{i_{2}}\left(t_{n}\right)$ is

$$
N_{i_{1}}<N_{i_{1}+1}<\cdots<N_{i}<\cdots<N_{i_{2}-1}<N_{i_{2}}
$$

which corresponds to $\left(i_{2}-i_{1}+1\right)$ levels. 
As in the classical multigrid methods, we use the concept of $\mathrm{V}$-cycle to improve the integration of (2.6) on the interval $\left[t_{n}, t_{n}+\right.$ Maxinc $\left.\triangle t\right]$. A multigrid cycle is divided on some subcycles (V-cycles) including each a phase of coming down and a phase of ascent.

(i) Phase of coming down. On the interval $\left[t_{n}, t_{n}+\left(i_{2}-i_{1}\right) \Delta t\right]$, the level $N_{i}(t)$ is defined by

$$
N_{i}(t)=N_{i_{2}-j}, \quad j=0, \ldots, i_{2}-i_{1}
$$

$N_{i}(t)$ decreases from $N_{i_{2}}\left(t_{n}\right)$ to $N_{i_{1}}\left(t_{n}\right)$.

(ii) Phase of ascent. On the interval $\left[t_{n}+\left(i_{2}-i_{1}\right) \triangle t, t_{n}+\left(2\left(i_{2}-i_{1}\right)+1\right) \triangle t\right]$, the level $N_{i}(t)$ is defined by

$$
N_{i}(t)=N_{i_{1}+\left(j-i_{2}+i_{1}-1\right)}, \quad j=i_{2}-i_{1}+1, \ldots, 2\left(i_{2}-i_{1}\right)+1,
$$

$N_{i}(t)$ increases from $N_{i_{1}}\left(t_{n}\right)$ to $N_{i_{2}}\left(t_{n}\right)$.

Then, a $\mathrm{V}$-cycle consists of $\left[2\left(i_{2}-i_{1}\right)+1\right]$ temporal iterations.

Maxinc is the maximal number of iterations to make a complete cycle (it is a multiple of $\left.\left[2\left(i_{2}-i_{1}\right)+1\right]\right)$. Let $t$ be an intermediate value in time on the interval $\left[t_{n}, t_{n}+\right.$ Maxinc $\left.\triangle t\right]$, then the current level $N_{i}(t)$ is given by

$$
N_{i}(t)= \begin{cases}N_{i_{2}-r+1}, & \text { si } 1 \leq r \leq i_{2}-i_{1}, \\ N_{i_{1}+\left(r-\left(i_{2}-i_{1}+1\right)\right)}, & \text { si } i_{2}-i_{1}+1 \leq r \leq 2\left(i_{2}-i_{1}\right),\end{cases}
$$

where $r$ is given by

$$
t-t_{n}=\left(2 p\left(i_{2}-i_{1}\right)+r\right) \triangle t .
$$

Knowing the size $N_{i}$ of the coarse level at the instant $t$, we decompose $u_{N}(t)$ into

$$
u_{N}(t)=y_{N_{i}}(t)+z_{N_{i}}(t)
$$

where $y_{N_{i}}(t)$ represents the large scales and $z_{N_{i}}(t)$ the small scales.

The computation of both components $y_{N_{i}}(t)$ and $z_{N_{i}}(t)$ are performed as follows.

(i) Computation of $z_{N_{i}}(t)$.

$$
z_{N_{i}}(t)=z_{N_{i}}(t-\triangle t)
$$

$z_{N_{i}}(t)$ is frozen and set to its last value, that is, its temporal variations are neglected.

(ii) Computation of $y_{N_{i}}(t)$. In order to evaluate $y_{N_{i}}(t)$, we integrate (2.12) over the interval $[t-\triangle t, t]$, then we obtain

$$
\begin{aligned}
\hat{y}_{k}(t)= & e^{-v|k|^{2} \Delta t} \hat{y}_{k}(t-\triangle t) \\
& +\int_{t-\triangle t}^{t} e^{-\nu|k|^{2}(t-\tau)}\left[\widehat{g}_{k}(\tau)-\widehat{B}_{k}\left(y_{N_{i}}(\tau), y_{N_{i}}(\tau)\right)\right] d \tau \\
& -\int_{t-\triangle t}^{t} e^{-\nu|k|^{2}(t-\tau)} \widehat{B}_{\mathrm{int}, k}\left(y_{N_{i}}(\tau), z_{N_{i}}(\tau)\right) d \tau
\end{aligned}
$$

for all $k \in I_{N_{i}}=\left[1-N_{i} / 2, N_{i} / 2\right]^{3}$. 

3.

The first integral of (3.13) is approached by an explicit Runge-Kutta scheme of order

With this scheme, the interval $[t-\triangle t, t]$ is divided into 3 subintervals of the form $\left[t_{i}, t_{i+1}\right]$ where $t_{0}=t-\triangle t$ and $t_{3}=t$. The second integral carrying on the terms of interactions is calculated by making a quasistatic approximation on these terms, which is equivalent to approaching this integral by an explicit Euler scheme of order 1 on the subintervals $\left[t_{i}, t_{i+1}\right], i=0,1,2$. At the end the cycle, that is, at $t=t_{n}+$ Maxinc $\Delta t, z_{N_{i_{2}}}(t)$ is revalued by projecting the solution on the approximate inertial manifold of the form

$$
z_{N_{i}}(t)=\phi\left(y_{N_{i}}(t)\right)
$$

for every intermediate level, $N_{i} \in\left[N_{i_{1}}, N_{i_{2}}\right]$, the coupling nonlinear terms $B_{\text {int }}\left(y_{N_{i}}, z_{N_{i}}\right)$ are frozen on the interval $\left[t_{n}, t_{n}+\right.$ Maxinc $\left.\triangle t\right]$, revalued then at the end of the cycle.

At the end of the cycle, we evaluate new values of the two levels $N_{i_{1}}$ and $N_{i_{2}}$ by the tests (3.4) and (3.5) and we start again the procedure.

The interaction between the different scales of the flow is taken then into account of simplified manner. We define 3 dynamical zones on the whole of the excited modes:

(1) a zone entirely included in the zone of dissipation named quasistatic zone $(k \geq$ $N_{i_{2}}$ ) defining the small scales frozen and then relaxed (these small scales and their interactions with the large ones are indeed negligible locally in time but not longterm);

(2) a transition zone $\left(N_{i_{1}} \leq k \leq N_{i_{2}}\right)$ or intermediate zone; use of a multigrid V-cycle strategy between the two levels $N_{i_{1}}$ and $N_{i_{2}}$ to assure a transition between a stationary approximation (zone (1)) and an integration in time with a time step $\triangle t$ (zone (3));

(3) dynamical zone defining the large scales $\left(k \leq N_{i_{1}}\right)$ calculated to every time step $\triangle t$ by a quadrature method for the exact integration of the linear part, by an explicit Runge-Kutta scheme of order 3 for the nonlinear term $B(y, y)$, and by one quasistationary approximation for the coupling nonlinear terms $B_{\text {int }}(y, z)$.

The multigrid methods permit to accelerate the convergence of an iterative method by obtaining the same precision that if one had only used the fine grid. In the same way, the nonlinear Galerkin method permits to accelerate the process of evolution, with the same precision of the classical Galerkin method (usual) to $N$ modes ( $N$ being the number of modes on the fine grid).

\section{Numerical results}

We compare the two methods: usual Galerkin (UG) and nonlinear Galerkin (NGL) on examples of which we know the exact solution $\left(u_{\mathrm{ex}}\right)$. We can compare then the precision of the two methods and the time CPU consumed by iteration in time.

\subsection{Example 1}

4.1.1. Simplified description of the example. The goal is to find a periodic solution in space of the equations of Navier-Stokes in dimension 3 having an energy spectrum with an inertial zone of slope $k^{-5 / 3}$ (Kolmogorov) with a predominant peak centered in one mode 
and a viscous zone to fast decrease of the energy. In spite of the fact that this type of solutions is artificial, these solutions present the structures of different sizes. We impose, moreover, that the small structures do not follow the same temporal evolution as the large structures.

Such example can be considered as one approach in three-dimensional turbulence.

We give the equation of Navier-Stokes:

$$
\frac{\partial u}{\partial t}-v \triangle u+B(u, u)=f
$$

$u$ is periodic in space in the 3 directions. We can decompose $u$ in Fourier series:

$$
u(x, t)=\sum_{k \in \mathbb{Z}^{3}} \hat{u}_{k}(t) e^{i k \cdot x} .
$$

For a fixed $N \in \mathbb{N}$, we define the truncated series

$$
u_{N}(x, t)=\sum_{k \in I_{N}} \widehat{u}_{k}(t) e^{i k \cdot x}
$$

where $I_{N}=[1-N / 2, N / 2]^{3}$ is a subspace of $\mathbb{Z}^{3} \cdot u_{N}=P_{N} u$ where $P_{N}$ is the operator of projection on the subspace spanned by $\left(e^{i k \cdot x}\right) k \leq N / 2 . u_{N}$ possesses energy spectrum $E(k, t)$ having the following form (see Figure 4.7):

$$
E(k, t)=\sum_{l=\left(k_{1}, k_{2}, k_{3}\right), k_{1}^{2}+k_{2}^{2}+k_{3}^{2}=k^{2}}\left|\hat{u}_{1, l}\right|^{2}+\left|\hat{u}_{2, l}\right|^{2}+\left|\hat{u}_{3, l}\right|^{2}, \quad k \in\left[1, \frac{N}{2}\right] .
$$

With the help of $E(k, t)$, we determine the coefficients of Fourier $\hat{u}_{k}$ of $u_{N}$.

So $P_{N} f$ is determined by

$$
P_{N} f=\frac{d u_{N}}{d t}-v \triangle+P_{N} B\left(u_{N}, u_{N}\right)
$$

The energy spectrum is written as

$$
\begin{aligned}
E(k, t) & =c_{1} k^{-5 / 3}, \quad k \leq K N_{1}, K N_{1}<\frac{N}{2}, \\
E\left(k_{F}, t\right) & =C_{F}(t) c_{1} k^{-5 / 3}, \quad k_{F} \leq K N_{1}, \\
E(k, t) & =G(k, t), \quad k>K N_{1}, K N_{1}<\frac{N}{2}, \\
G(k, t) & =\exp \left[\log (10) g\left(\log _{10}(k), t\right)\right],
\end{aligned}
$$

where

$$
g(x, t)=g\left(x_{1}, t\right)-\frac{5}{3}\left(x-x_{1}\right) e^{\alpha(t)\left(x-x_{1}\right)}
$$


(with $G\left(K N_{1}, t\right)=E\left(K N_{1}, t\right), g$ function of class $\left.C^{1}\right)$,

$$
\begin{gathered}
x_{1}=\log _{10}\left(K N_{1}\right), \\
x_{2}=\log _{10}\left(K N_{2}\right), \\
\alpha(t)=\frac{1}{x_{2}-x_{1}} \log \left(\frac{3}{5} \frac{\log _{10}\left(C_{2}(t)\right)+g\left(x_{1}, t\right)}{x_{2}-x_{1}}\right), \\
E\left(K N_{2}, t\right)=G\left(K N_{2}, t\right)=C_{2}(t),
\end{gathered}
$$

$C_{2}$ is one bounded function $\left(C_{2} \in\left[c_{2 \min }, c_{2 \max }\right]\right)$.

In this example,

$$
\begin{aligned}
C_{F}(t)=\frac{1}{10}( & \cos (t) \sin (\sqrt{2} t)+2.5 \exp \left(-0.5(t-5)^{2}\right) \\
& \left.+\exp \left(-0.25(t-7)^{2}\right)+\frac{\sin (2 \sqrt{7} t)}{5}\right)+1,
\end{aligned}
$$

$C_{2}(t)$ is determined by

$$
\begin{gathered}
F(t)=\sum_{k=1}^{15} \exp \left(\cos \left(\frac{2 \pi}{10}(k \sqrt{k}(2.5+0.25 t))\right)-0.3 \sin \left(0.80 \frac{2 \pi}{10} k \sqrt{k} t\right)\right) \\
+10 \exp \left(-(t-5)^{2}\right), \\
F_{\text {min }}=\min (F(t)) \text { and } F_{\text {max }}=\max (F(t)) ; \\
\operatorname{Dev}=\frac{c_{2 \max }-c_{2 \min }}{F_{\max }-F_{\min }}, \\
C_{2}(t)=c_{2 \min }+\operatorname{Dev}\left(F(t)-F_{\min }\right),
\end{gathered}
$$

$C_{F}(t)$ contributes to the evolution of the low modes of the solution. $C_{2}(t)$ contributes to the evolution of the high modes of the solution.

\subsubsection{Significance of the parameters.}

(i) $\triangle t$ denotes time step $\left(=0.2510^{-2}\right)$.

(ii) $v$ denotes viscosity $\left(=0.110^{-1}\right)$.

(iii) $N$ denotes the total number of modes used for the spectral discretisation in space $(=48)$, that is, $48^{3}$ unknown.

(iv) Tol0 denotes parameter intervening in the multigrid strategy to define the minimal level of the coarse grids $N_{i_{1}}\left(=0.210^{-6}\right)$.

(v) Tol 1 denotes parameter intervening in the multigrid strategy to define the maximal level $N_{i_{2}}$ of the coarse grids during a cycle $\left(=0.210^{-8}\right)$. 


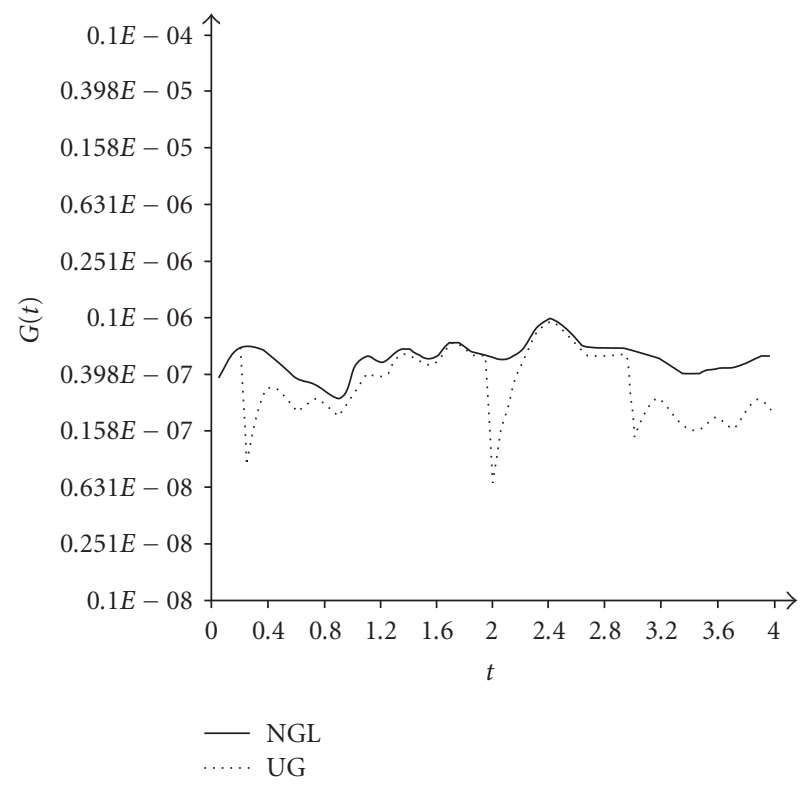

Figure 4.1. Relative error in norm $L^{2}\left(\left\|u-u_{\mathrm{ex}}\right\|_{L^{2}} /\left\|u_{\mathrm{ex}}\right\|_{L^{2}}\right)$.

(vi) Maxinc denotes parameter fixing the number of temporal iterations of a multigrid cycle.

(vii) $K N_{1}$ denotes parameter in the energy spectrum defining the solution $(=4)$.

(viii) $K N_{2}$ denotes parameter in the energy spectrum defining the solution (=12).

(ix) $c_{1}$ denotes parameter in the spectrum $(=5)$.

(x) $c_{2 \min }$ and $c_{2 \max }$ denote the bounds of the temporal function $C_{2}\left(=0.510^{-5}\right.$ and $\left.0.510^{-4}\right)$.

4.1.3. Commentary on the figures. Figures 4.1 and 4.2 compare the precision of the two methods: UG and NGL. Figure 4.1 measures the relative error in norm $\mathbf{L}^{2}$, that is, globally in the whole domain. Figure 4.2 measures the relative error in norm $\mathbf{L}^{\infty}$, that is, locally. As we can note, the precision obtained with NGL remains near the one obtained with UG. Figure 4.3 shows that the gain of computing time of NGL in comparison with UG is between $20 \%$ and $30 \%$, we can explain this by the fact that we calculate less often the coefficients associated to the elevated modes by using coarse grids on which are only valued the coefficients associated to the small modes.

Figure 4.4 represents the evolution of CPU time consumed in seconds for UG and NGL and justify the gain of computing time of NGL in comparison with UG.

The method NGL permits to get a better numerical stability than the method UG; the coefficients associated to the most elevated modes being the smallest. It is in the calculation of these coefficients that the relative errors made are the biggest. Contrary to the method UG, in not valuing the coefficients associated to the raised modes to every step of time, the method NGL permits to avoid the accumulation on every step of time of the errors made in the calculation of these coefficients, improving the stability. 


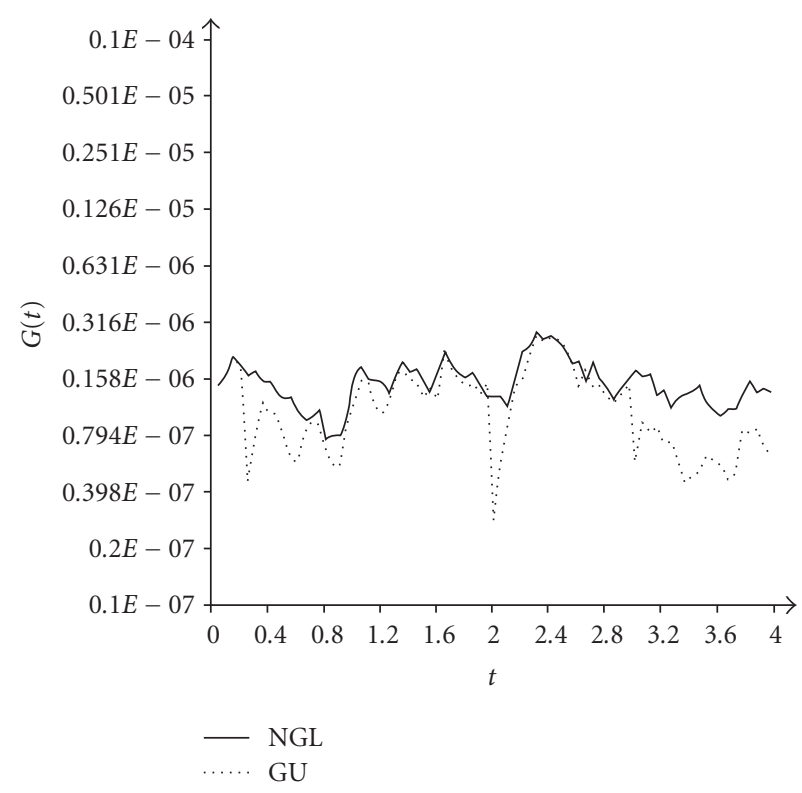

Figure 4.2. Relative error in norm $L^{\infty}\left(\left\|u-u_{\mathrm{ex}}\right\|_{L^{\infty}} /\left\|u_{\mathrm{ex}}\right\|_{L^{\infty}}\right)$.

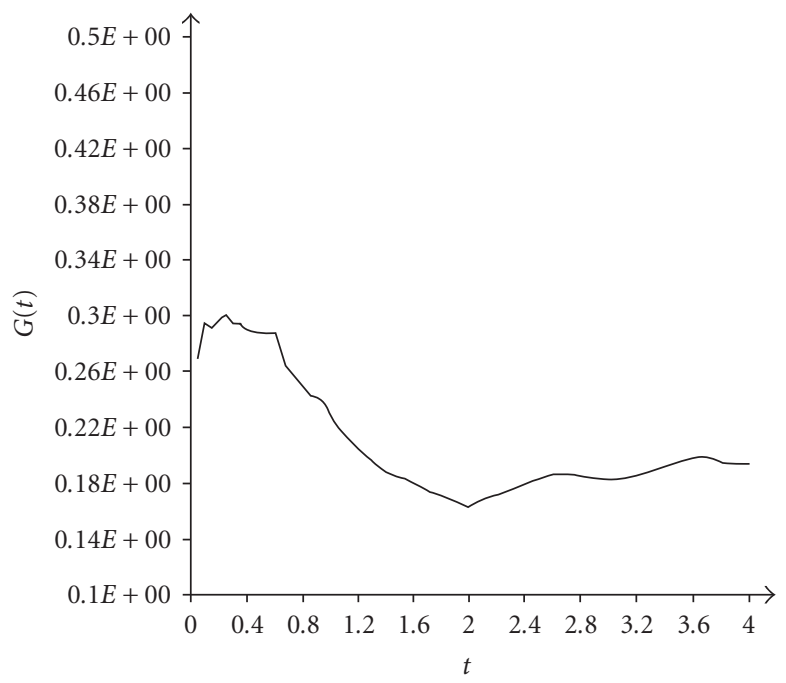

Figure 4.3. Gain of computing time for NGL over UG.

Figure 4.5 shows the evolution of the lower envelope of the levels of coarse grids $\left(N_{i_{1}}\right)$ with the method NGL, and Figure 4.6 represents the time evolution of the ratio $\|z\|_{L^{2}} /$ $\|y\|_{L^{2}}$ on this lower envelope. This ratio remains lower than $0.210^{-6}$ which is Tol0. It justifies the quasistatic approximation of the raised modes and of the terms of interactions. 
352 Multilevel method of nonlinear Galerkin type

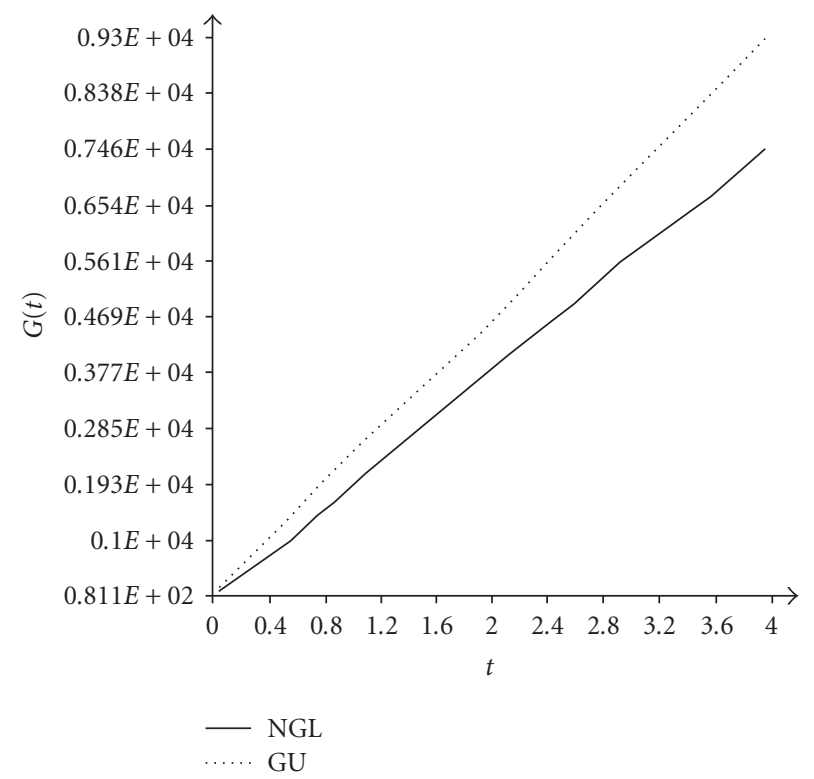

Figure 4.4. Comparison of CPU times (in seconds) by NGL and UG.

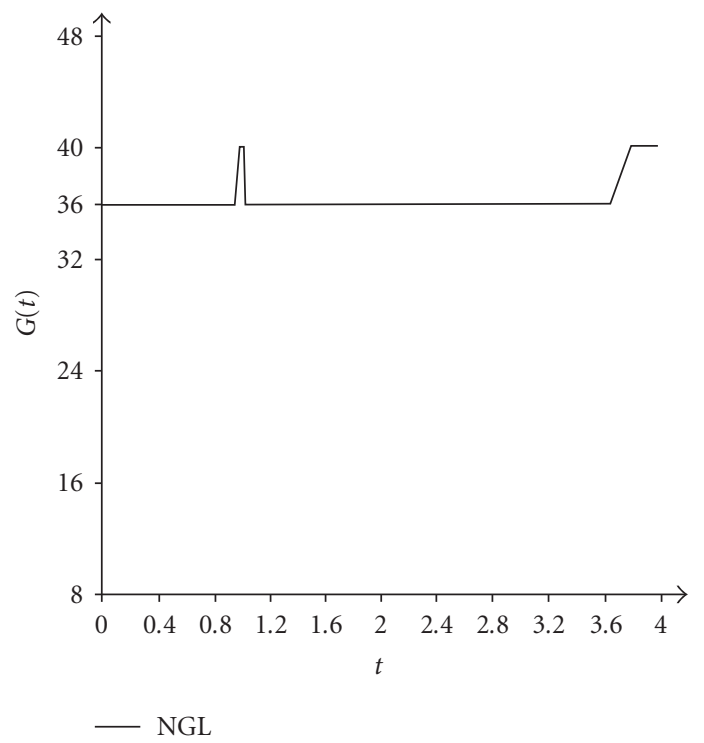

Figure 4.5. Lower envelope of the levels of discretization.

The abrupt oscillations of this ratio correspond to the changes of levels of the lower envelope. 


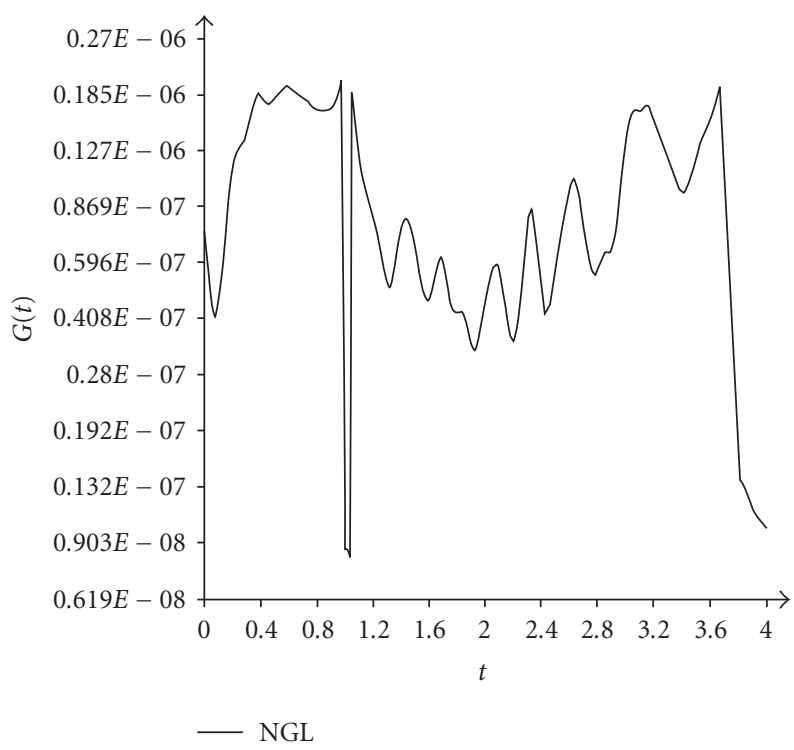

Figure 4.6. Time evolution of $\|z\|_{L^{2}} /\|y\|_{L^{2}}$ on the lower envelope.

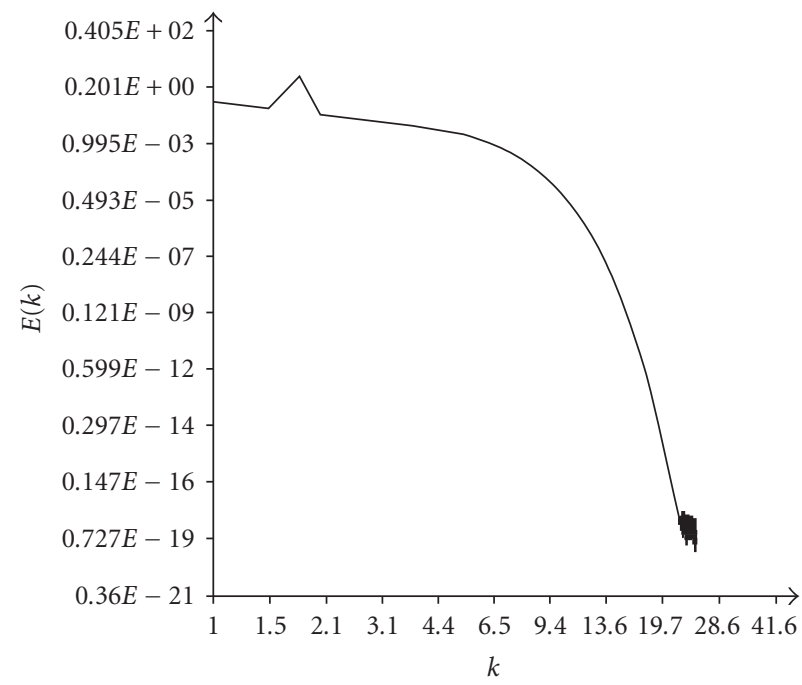

Figure 4.7. Energy spectrum at $t=4.00$ by NGL.

Figure 4.7 represents the energy spectrum of the solution calculated with NGL at $t=4$; we note that this spectrum is in conformity with the data of the exact solution.

The vorticity plays an important role in the generation of small scales in space driving to the turbulence. Figures 4.8 and 4.9 represent the isovorticity lines calculated with NGL 
354 Multilevel method of nonlinear Galerkin type

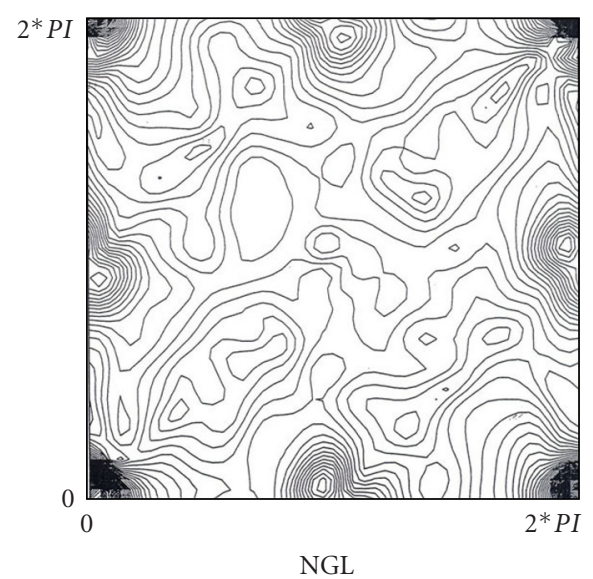

Figure 4.8. Isovorticity lines at $t=0.0$ by NGL.

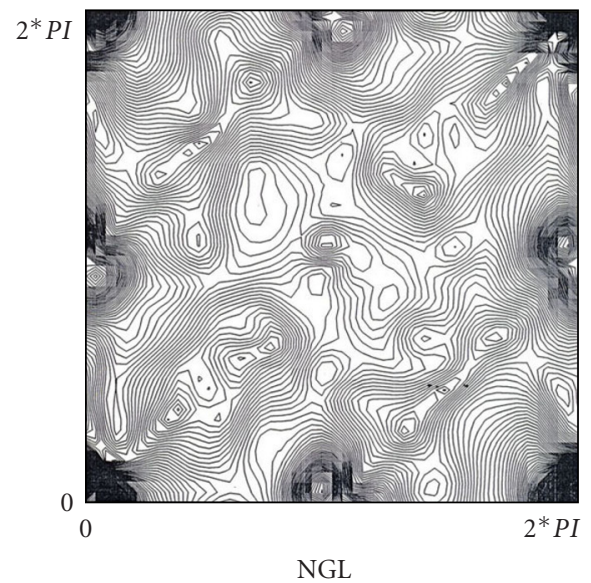

Figure 4.9. Isovorticity lines at $t=4.0$ by NGL.

at $t=0$ and at $t=4$ on the plan $z=\pi$, we notice the distortion of the large structures under the action of other large structures and of smaller structures. This distortion drives to the shearing of these large deformed structures and to the creation of new smaller structures that finish by disappearing. Large structures are created by the action of external force. The large structures that occupy the corners of the domain are directly supplied by external force.

\subsection{Example 2}

$$
g(t)=\frac{1}{10}(\cos (4.8 t)+\cos (3.2 \pi t)+0.5 \exp (3 \sin (1.6 t))+3)
$$




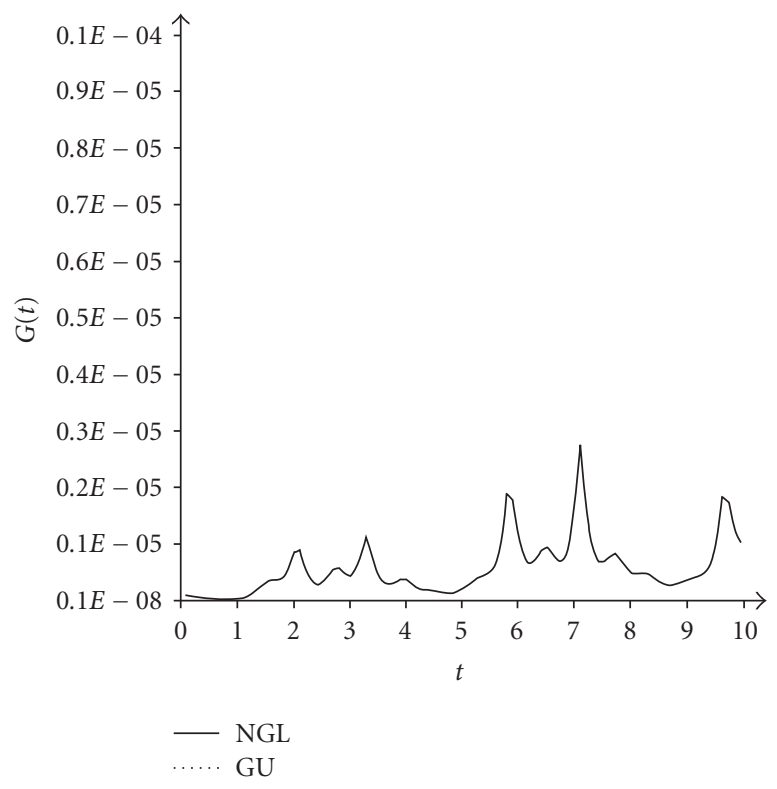

Figure 4.10. Relative error in norm $L^{2}\left(\left\|u-u_{\mathrm{ex}}\right\|_{L^{2}} /\left\|u_{\mathrm{ex}}\right\|_{L^{2}}\right)$.

The exact solution $u_{\mathrm{ex}}=\left(u_{1}, u_{2}, u_{3}\right)$,

$$
\begin{aligned}
& u_{1}=g(t) \exp (\cos y), \\
& u_{2}=g(t) \exp (\cos z), \\
& u_{3}=g(t) \exp (\cos x) .
\end{aligned}
$$

The external force is written as

$$
f(x, t)=\frac{d u_{\mathrm{ex}}}{d t}-v \triangle u_{\mathrm{ex}}+P_{N} B\left(u_{\mathrm{ex}}, u_{\mathrm{ex}}\right),
$$

the number of modes $N=24$, that is, $24^{3}$ unknown, $\triangle t$ time step $=5.10^{-3}$, and the viscosity $v=10^{-2}$.

Figures 4.10 and 4.11 measure the relative error in norms $L^{2}$ and $L^{\infty}$ for UG and NGL. The curves obtained with UG and NGL are practically identical: the precisions obtained by the two methods are practically the same.

Figure 4.12 shows the gain of computing time of NGL in comparison with UG which is the order of $30 \%$.

Figure 4.13 shows the evolution of CPU time consumed in seconds for UG and NGL.

Figures 4.14 and 4.15 represent the time evolution, respectively, of the quantities $\|y\|_{L^{2}}$ for UG on different levels and for $\|z\|_{L^{2}}$.

Figures 4.16 and 4.17 show the evolution of $y$ and $z$ during the time in norm $L^{2}$ with NGL. We can compare these figures with Figures 4.14 and 4.15 , we note that the time evolutions of $y$ in these figures for UG and NGL are identical (convergence of $u_{N}$ ). 
356 Multilevel method of nonlinear Galerkin type

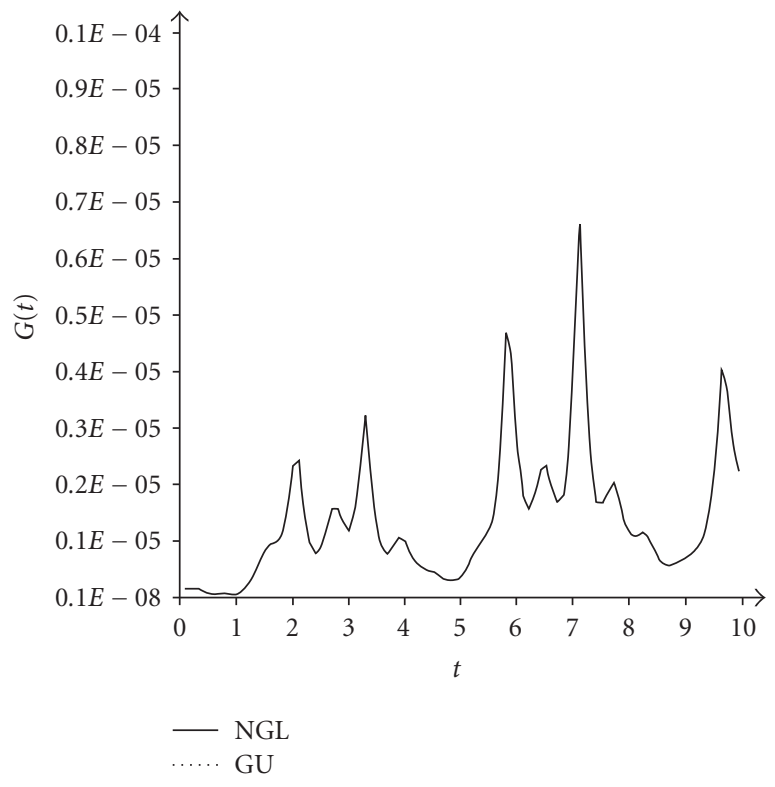

Figure 4.11. Relative error in norm $L^{\infty}\left(\left\|u-u_{\mathrm{ex}}\right\|_{L^{\infty}} /\left\|u_{\mathrm{ex}}\right\|_{L^{\infty}}\right)$.

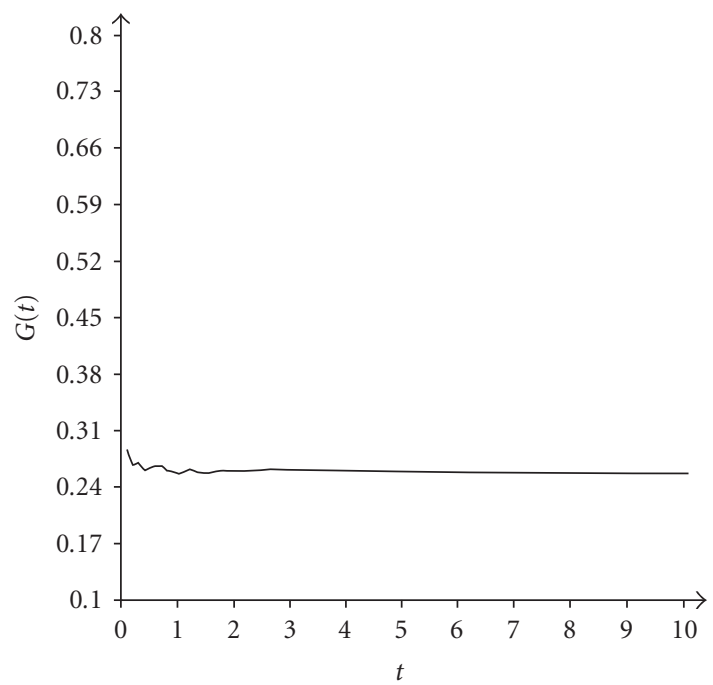

Figure 4.12. Gain of computing time for NGL over UG.

For the evolution of $z$, for $N 1=12$ and 16 , UG and NGL are practically identical whereas for the level 20, the difference between $z_{\mathrm{NGL}}$ and $z_{\mathrm{UG}}$ remains lower to the precision of the time scheme. 


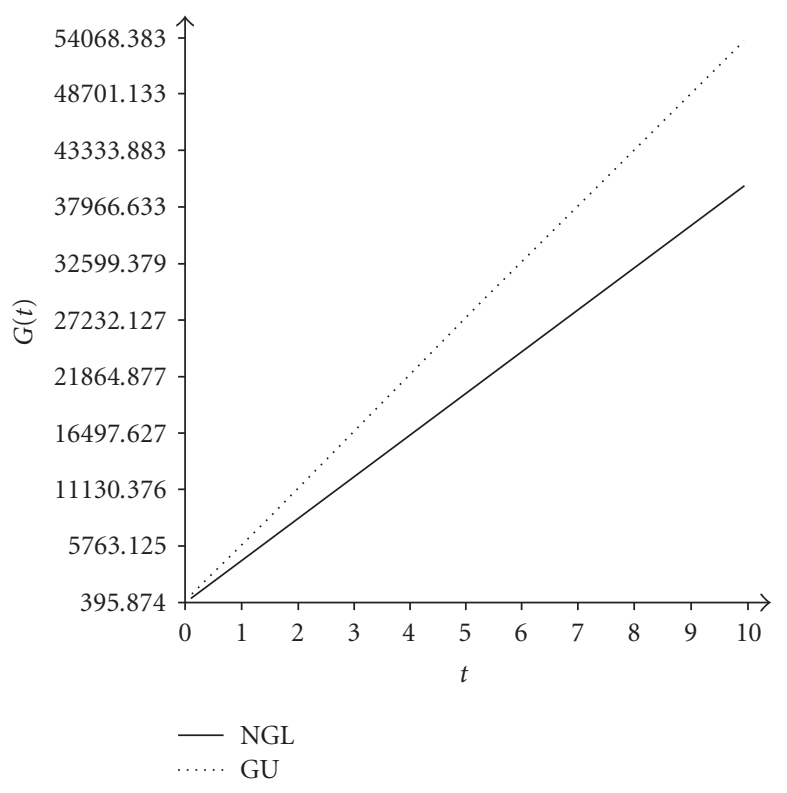

Figure 4.13. Comparison of CPU times (in seconds) by NGL and UG.

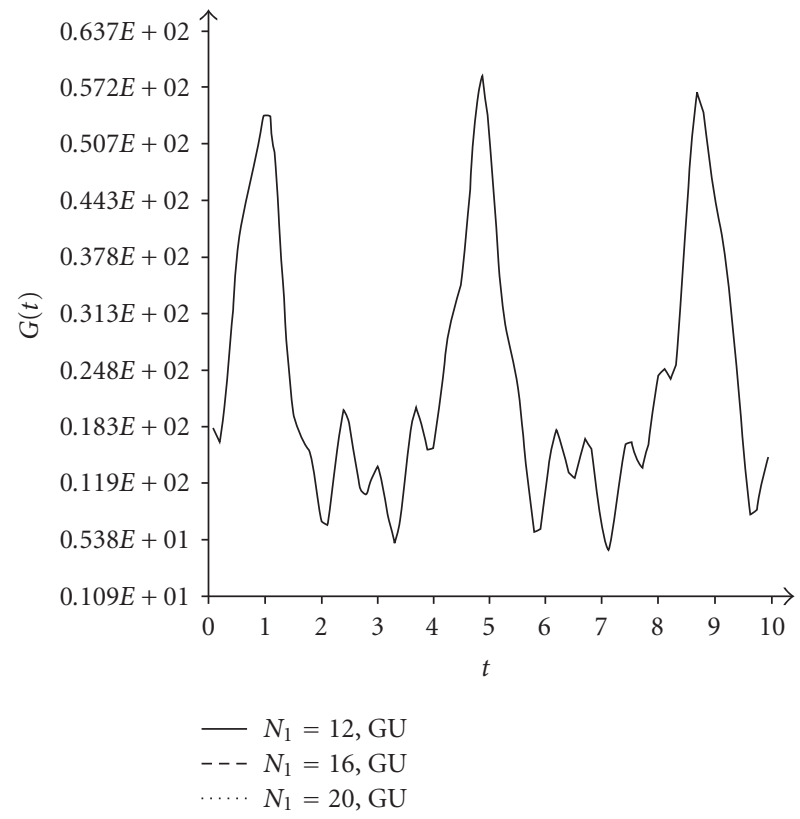

Figure 4.14. Time evolution of $\left\|y_{N_{1}}\right\|_{L^{2}}$ for $N_{1}=12,16$, and 20 by UG. 
358 Multilevel method of nonlinear Galerkin type

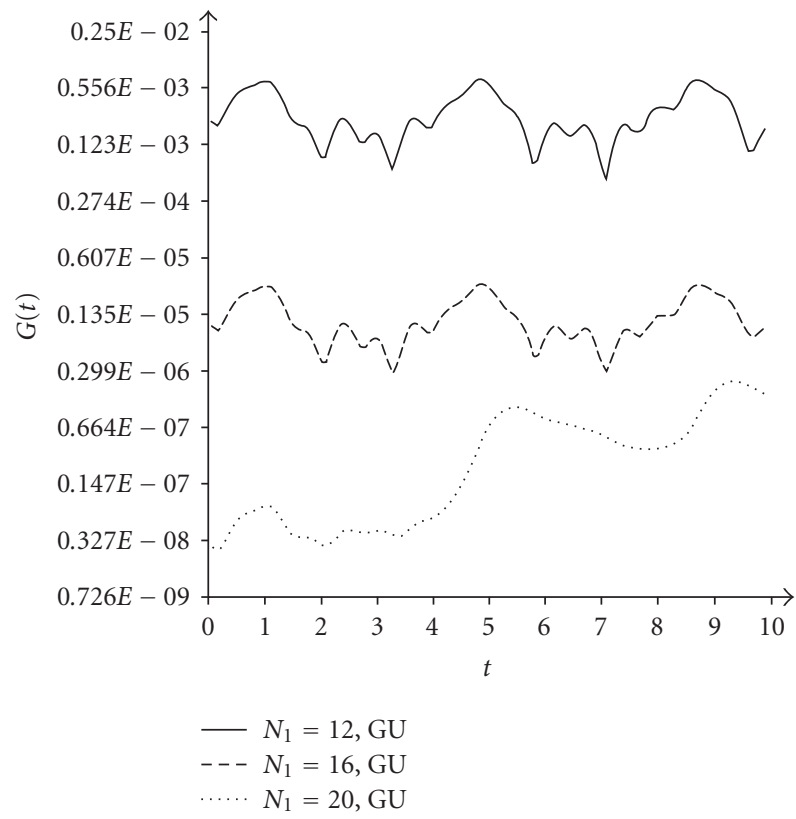

Figure 4.15. Time evolution of $\left\|z_{N_{1}}\right\|_{L^{2}}$ for $N_{1}=12,16$, and 20 by UG.

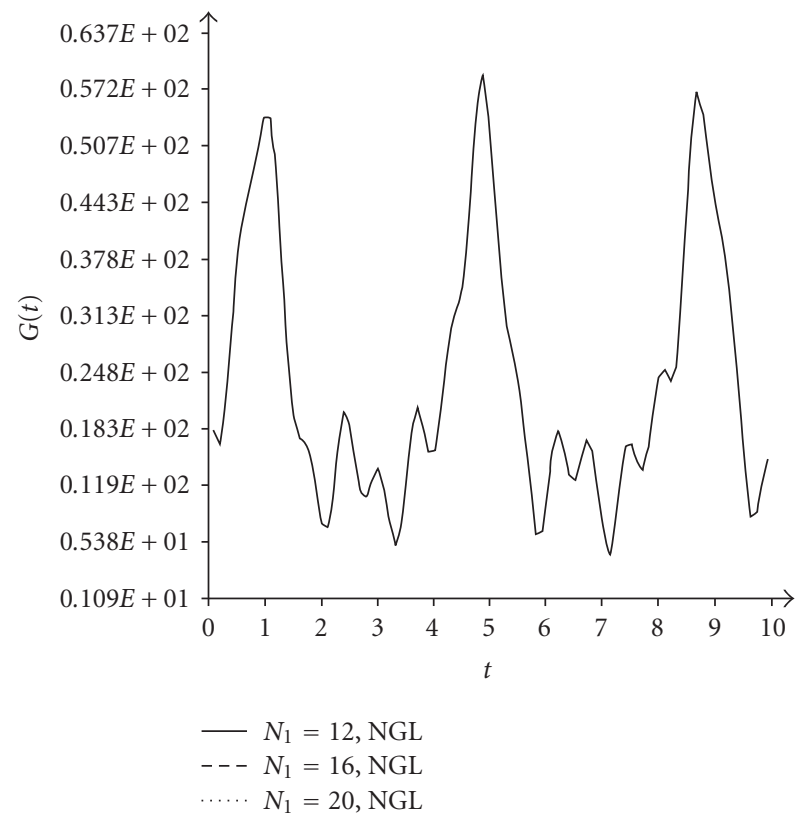

Figure 4.16. Time evolution of $\left\|y_{N_{1}}\right\|_{L^{2}}$ for $N_{1}=12,16$, and 20 by NGL. 


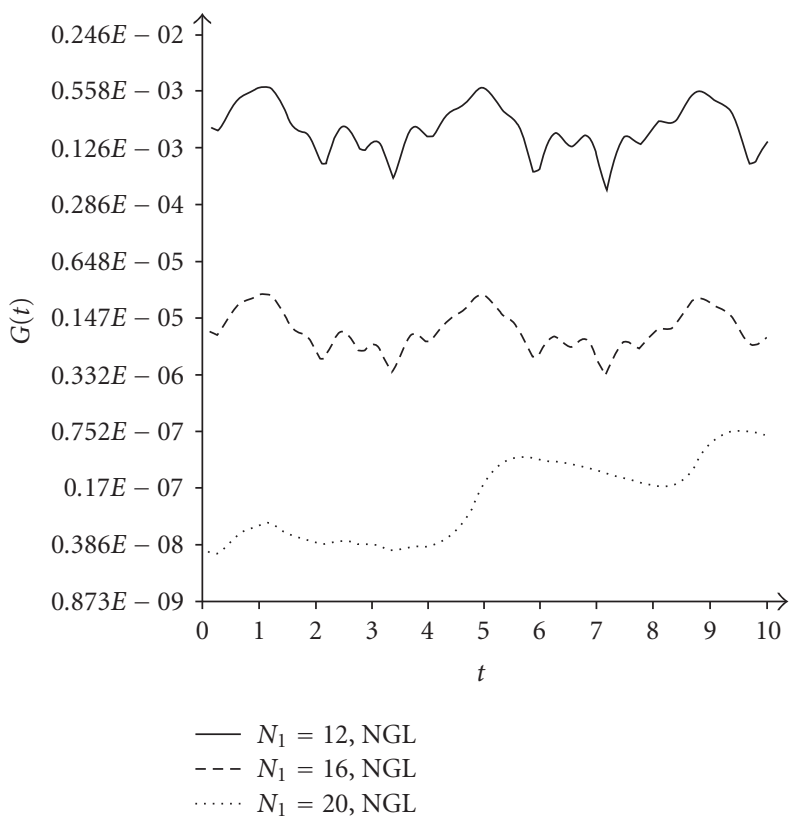

Figure 4.17. Time evolution of $\left\|z_{N_{1}}\right\|_{L^{2}}$ for $N_{1}=12,16$, and 20 by NGL.

\section{Stability analysis and estimation}

In [10], Jauberteau find again the CFL stability condition done in [7], for nonlinear Galerkin method:

$$
\triangle t N\left|u_{N}\right|_{L^{\infty}}<\alpha \quad \text { with } \alpha<1
$$

In [15], Temam describes some numerical schemes for the approximation of nonlinear evolution equations, in particular, Navier-Stokes equations, and studies the stability of the schemes, in particular, nonlinear Galerkin schemes.

We introduce two norms in $L^{2}$ and $H^{1}$ :

$$
\begin{aligned}
& |\varphi|_{2}=\left(\int_{\Omega}|\varphi(x, t)|^{2} d x\right)^{1 / 2}, \\
& \|\varphi\|=\left(\int_{\Omega}|\nabla \varphi(x, t)|^{2} d x\right)^{1 / 2}
\end{aligned}
$$

for any given field $\varphi(x, t)=\left(\varphi_{1}(x, t), \varphi_{2}(x, t), \varphi_{3}(x, t)\right)$,

$$
\Omega=\prod_{i=1}^{i=3}(0,2 \pi)
$$


We now aim to derive an estimate of the variations of $z_{N_{1}}$ over one time iteration. This quantity can be represented by

$$
\triangle_{N_{1}}=\triangle t\left|\dot{z}_{N_{1}}\right|_{2}
$$

where the dot represents the differentiation with respect to $t$. The time step $\Delta t$ is given by the CFL stability condition [7]:

$$
\triangle t N\left|u_{N}\right|_{L^{\infty}}<\alpha \quad \text { with } \alpha<1 .
$$

We recall that $N$ is the total number of modes in each direction. We assume that the smallest scale $l_{N}=1 / k_{N}, k_{N}=N / 2$ is smaller than the Kolmogorov dissipation scale $l_{\eta}=$ $1 / k_{\eta}$. We first consider the case where $l_{N_{1}}=1 / k_{N_{1}}$ is lying between $l_{\eta}$ and $l_{N}\left(l_{N} \leq l_{N_{1}} \leq l_{\eta}\right)$.

The time derivative $\dot{z}_{N_{1}}$ is of the order of the dissipation term (remember we are in the dissipation range):

$$
\left|\dot{z}_{N_{1}}\right|_{2} \sim v\left|\triangle z_{N_{1}}\right|_{2}
$$

Due to the exponential decay of the velocity spectrum in the dissipation range, we can write

$$
\nu\left|\triangle z_{N_{1}}\right|_{2} \leq c_{1} \nu k_{N_{1}}^{2}\left|z_{N_{1}}\right|_{2}
$$

where $c_{1}$ is a nondimensional constant of order of unity.

We obtain

$$
\triangle_{N_{1}} \leq c_{2} \frac{|g|_{2}^{1 / 3}}{\left|u_{N}\right|_{L^{\infty}}}\left(\frac{k_{N_{1}}}{k_{\eta}}\right) v^{1 / 3}\left|z_{N_{1}}\right|_{2} .
$$

Then, for sufficiently small values of the viscosity $\nu$, the variations of $z_{N_{1}}$ over one time iteration are much smaller than $\left|z_{N_{1}}\right|_{2}$.

We now try to derive a similar estimate when $l_{N_{1}}$ is in the inertial range, that is, $\left(l_{\eta} \leq\right.$ $l_{N_{1}}$ ). In that case, the time derivative $\dot{z}_{N_{1}}$ is of the order of the interaction nonlinear terms:

$$
\left|\dot{z}_{N_{1}}\right|_{2} \sim\left|Q_{N_{1}} B_{\text {int }}\left(y_{N_{1}}, z_{N_{1}}\right)\right|_{2}
$$

As $Q_{N_{1}}$ is a projection operator, we have

$$
\left|Q_{N_{1}} B\left(y_{N_{1}}, z_{N_{1}}\right)\right|_{2} \leq\left|B\left(y_{N_{1}}, z_{N_{1}}\right)\right|_{2} .
$$

The bilinear form $B$ can be estimated as follows (see, e.g., $[13,14]$ ):

$$
\left|B\left(y_{N_{1}}, z_{N_{1}}\right)\right|_{2} \leq c_{4}\left|y_{N_{1}}\right|_{L^{\infty}}|| z_{N_{1}}||
$$

The decay of the velocity Fourier components implies that

$$
\left\|z_{N_{1}}\right\| \leq c_{5} k_{N_{1}}\left|z_{N 1}\right|_{2}, \quad \text { where } c_{5} \sim 1,
$$


so that

$$
\left|Q_{N_{1}} B\left(y_{N_{1}}, z_{N_{1}}\right)\right|_{2} \leq c_{4} c_{5} k_{N_{1}}\left|y_{N_{1}}\right|_{L^{\infty}}\left|z_{N_{1}}\right|_{2} .
$$

From inequality (5.7), we deduce that

$$
\left|Q_{N_{1}} B\left(z_{N_{1}}, y_{N_{1}}\right)\right|_{2} \leq c_{8} k_{N_{1}}\left|y_{N_{1}}\right|_{L^{\infty}}\left|z_{N_{1}}\right|_{2} .
$$

A similar estimate can be derived for the third term $Q_{N_{1}} B\left(z_{N_{1}}, z_{N_{1}}\right)$.

We finally obtain

$$
\left|Q_{N_{1}} B_{\text {int }}\left(y_{N_{1}}, z_{N_{1}}\right)\right|_{2} \leq c_{9} k_{N_{1}}\left|u_{N}\right|_{L^{\infty}}\left|z_{N_{1}}\right|_{2}
$$

Then,

$$
\triangle_{N_{1}} \leq c_{9} k_{N_{1}} \triangle t\left|u_{N_{1}}\right|_{L^{\infty}}\left|z_{N_{1}}\right|_{2}
$$

As

$$
\triangle t \leq \frac{\alpha}{N\left|u_{N}\right|_{L^{\infty}}},
$$

we find

$$
\triangle_{N_{1}} \leq c_{9} \frac{\alpha}{\sqrt{2}} \frac{k_{N_{1}}}{k_{N}}\left|z_{N_{1}}\right|_{2}
$$

which also implies that $\triangle_{N_{1}}<\left|z_{N_{1}}\right|_{2}$ in the inertial range as well. For more details on such developments, the reader is referred to Dubois [4].

In [3], Devulder et al. provide estimates to the rate of the convergence of the nonlinear Galerkin method, they show that the nonlinear Galerkin converges faster than the usual Galerkin method as in the numerical examples.

We present here two theorems which give an error estimate in the norm of $H$ for the usual Galerkin method and nonlinear Galerkin method.

TheOREM 5.1. Let $u(t)$ be a solution of (2.6) such that

$$
\|u(t)\| \leq M_{1} \quad \forall t \geq 0
$$

Suppose that $y_{m}(t)$ is the truncated solution of the system (2.6) as for the usual Galerkin method, that is, without separation of the scales which satisfies $y_{m}(0)=P_{m} u_{0}$ and

$$
\left\|y_{m}(t)\right\| \leq M_{1} \quad \forall t \geq 0
$$

Then

$$
\begin{aligned}
& \left|u(t)-y_{m}(t)\right|^{2} \leq\left(\int_{0}^{t} e^{\int_{s}^{t} A_{m}(\tau) d \tau} B_{m}(s) d s+\frac{2}{L_{m}}\right) \frac{L_{m}^{2}}{\lambda_{m+1}^{2}} \\
& \left\|u(t)-y_{m}(t)\right\|^{2} \leq\left(2+\int_{0}^{t} e^{\int_{s}^{t} \widetilde{A}_{m}(\tau) d \tau} \widetilde{B}_{m}(s) d s\right) \frac{L_{m}}{\lambda_{m+1}}
\end{aligned}
$$


where $L_{m}=1+\log \left(\lambda_{m} / \lambda_{1}\right), \lambda_{m}$ denotes the $m$ th eigenvalue of the stokes operator $-\triangle . A_{m}(t)$ and $B_{m}(t)$ are variables in time depending on $t,\|u\|,\|y\|$.

For the calculation of these variables and the proof, see [3].

Theorem 5.2. Let $u(t)$ be a solution of (2.6) such that

$$
\|u(t)\| \leq M_{1} \quad \forall t \geq 0 .
$$

Suppose that $y_{m}(t)+\phi\left(y_{m}(t)\right)$ is the truncated solution of the system (2.12), (2.13) which satisfies $y_{m}(0)=P_{m} u_{0}$ and

$$
\left\|y_{m}(t)\right\| \leq M_{1} \quad \forall t \geq 0
$$

Then

$$
\begin{aligned}
\left|u(t)-\left(y_{m}(t)+\phi\left(y_{m}(t)\right)\right)\right|^{2} & \leq\left[\left(1+\frac{K}{\lambda_{m+1}^{1 / 2}}\right) \int_{0}^{t} e^{\int_{s}^{t} A_{m}(\tau) d \tau} B_{m}(s) d s+\frac{2}{L_{m}}\right] \frac{K L_{m}^{3}}{\lambda_{m+1}^{3}}, \\
\left\|u(t)-\left(y_{m}(t)+\phi\left(y_{m}(t)\right)\right)\right\|^{2} & \leq\left[\left(1+\frac{K}{\lambda_{m+1}^{1 / 4}}\right) \int_{0}^{t} e^{\int_{s}^{t} \widetilde{A}_{m}(\tau) d \tau} \widetilde{B}_{m}(s) d s+2\right] \frac{K L_{m}^{2}}{\lambda_{m+1}^{2}}
\end{aligned}
$$

where $L_{m}=1+\log \left(\lambda_{m} / \lambda_{1}\right), \lambda_{m}$ denotes the mth eigenvalue of the stokes operator $-\triangle . \tilde{A}_{m}(t)$ and $\widetilde{B}_{m}(t)$ are variables in time depending on $t,\|u\|,\|y\|$ and $\phi$.

For the calculation of these variables and the proof, see [3].

See also Heywood and Rannacher [9] for other estimations.

\section{Conclusion}

The numerical tests that we have done showed significant gain in computing time of this method in comparison with the usual Galerkin method and kept a comparable precision to this last one.

This new scheme of resolution permits a simplification of the calculation of the coefficients associated to the elevated modes of the solution and keeps the precision of their interaction with the coefficients associated to smaller modes.

The large precision of the spectral methods is kept.

This new scheme is well adapted to the resolution of the evolutionary problems on large intervals of time.

\section{References}

[1] W. L. Briggs, A Multigrid Tutorial, Society for Industrial and Applied Mathematics (SIAM), Pennsylvania, 1987.

[2] C. Canuto, M. Y. Hussaini, A. Quarteroni, and T. A. Zang, Spectral Methods in Fluid Dynamics, Springer Series in Computational Physics, Springer, New York, 1988.

[3] C. Devulder, M. Marion, and E. S. Titi, On the rate of convergence of the nonlinear Galerkin methods, Math. Comp. 60 (1993), no. 202, 495-514.

[4] T. Dubois, Simulation numérique d'écoulements homogènes et non homogènes par des méthodes de multi-résolution, Thesis, Universite de Paris XI, Orsay, 1993. 
[5] T. Dubois, F. Jauberteau, and R. Temam, Dynamics multilevel methods and turbulence, Encyclopedia of Computational Mechanics (E. Stein, R. de Borst, and T. Jr. Hughes, eds.), John Wiley, 2004.

[6] C. Foias, O. Manley, and R. Temam, Modelling of the interaction of small and large eddies in twodimensional turbulent flows, RAIRO Modél. Math. Anal. Numér. 22 (1988), no. 1, 93-118.

[7] S. Godunov and V. Riabenki, Schémas aux Différences, Éditions Mir, Moscow, 1977 (French).

[8] W. Hackbusch, Multigrid Methods and Applications, Springer Series in Computational Mathematics, vol. 4, Springer, Berlin, 1985.

[9] J. G. Heywood and R. Rannacher, On the question of turbulence modeling by approximate inertial manifolds and the nonlinear Galerkin method, SIAM J. Numer. Anal. 30 (1993), no. 6, 16031621.

[10] F. Jauberteau, Résolution numérique des équations de Navier-Stokes instationnaires par méthodes spectrales. Méthode de Galerkin non linéaire, Thesis, Universite de Paris XI, Orsay, 1990.

[11] F. Jauberteau, C. Rosier, and R. Temam, A nonlinear Galerkin method for the Navier-Stokes equations, Comput. Methods Appl. Mech. Engrg. 80 (1990), no. 1-3, 245-260.

[12] M. Marion and R. Temam, Nonlinear Galerkin methods, SIAM J. Numer. Anal. 26 (1989), no. 5, $1139-1157$.

[13] R. Temam, Navier-Stokes Equations. Theory and Numerical Analysis, North-Holland Publishing Company, Amsterdam, 1977.

[14] - Navier-Stokes Equations and Nonlinear Functional Analysis, CBMS-NSF Regional Conference Series in Applied Mathematics, vol. 41, Society for Industrial and Applied Mathematics (SIAM), Pennsylvania, 1983.

[15] Stability analysis of the nonlinear Galerkin method, Math. Comp. 57 (1991), no. 196, 477-505.

Said El Hajji: Department of Mathematics and Informatics, Faculty of Sciences, University Mohammed V-Agdal, B.P. 1014, Rabat, Morocco

E-mail address: elhajji@fsr.ac.ma

Khalid Ilias: Department of Mathematics and Informatics, Faculty of Sciences, University Mohammed V-Agdal, B.P. 1014, Rabat, Morocco

E-mail address: ilias_khalid@yahoo.fr 


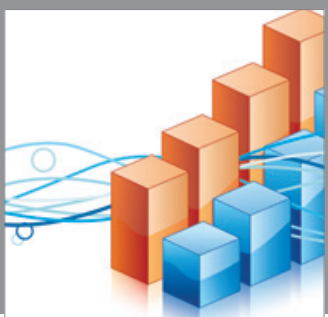

Advances in

Operations Research

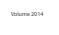

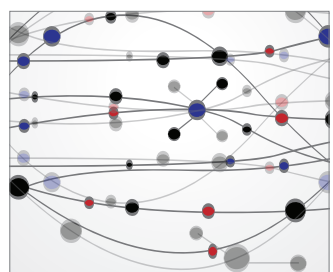

\section{The Scientific} World Journal
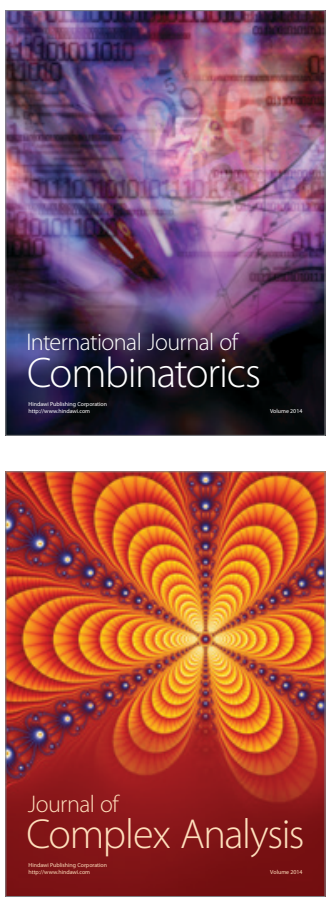

International Journal of

Mathematics and

Mathematical

Sciences
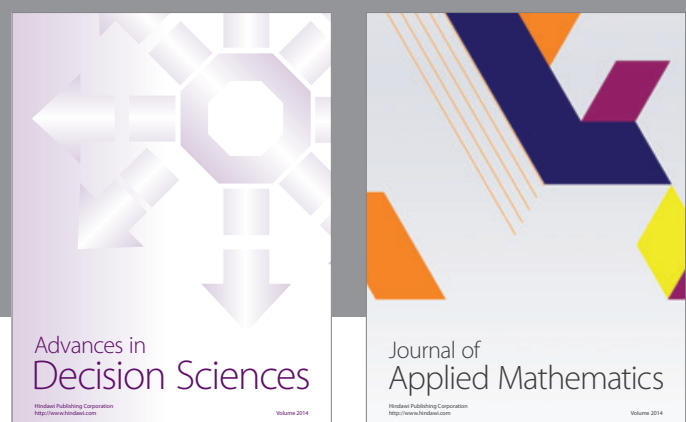

Journal of

Applied Mathematics
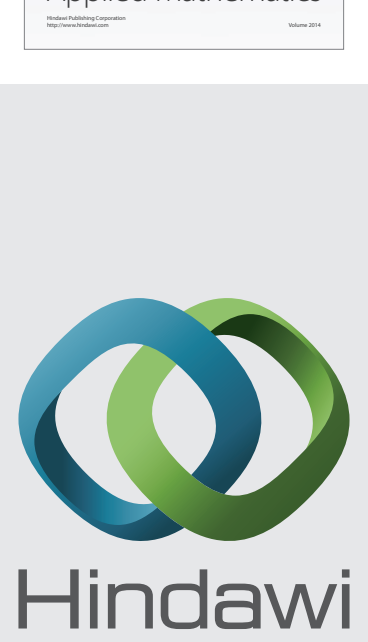

Submit your manuscripts at http://www.hindawi.com
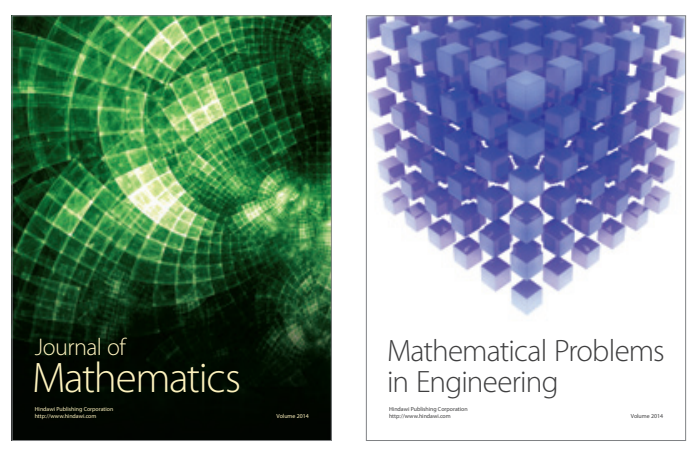

Mathematical Problems in Engineering
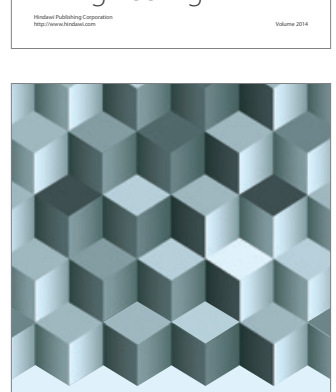

Journal of

Function Spaces
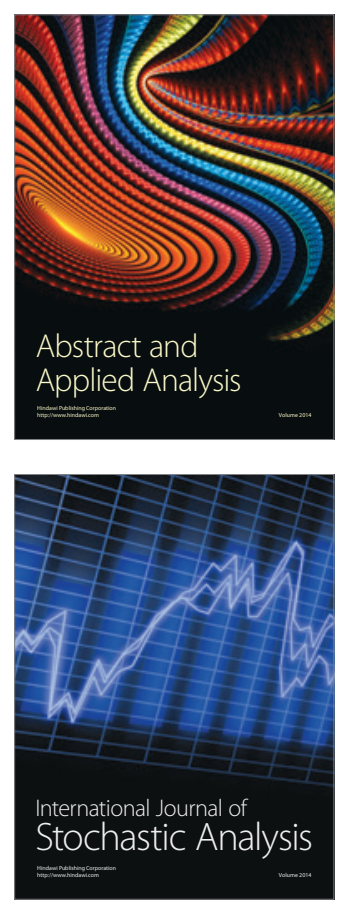

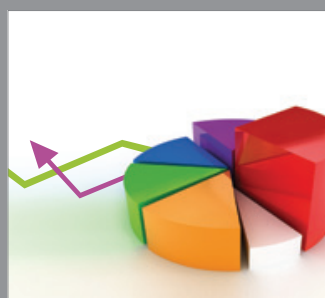

ournal of

Probability and Statistics

Promensencen
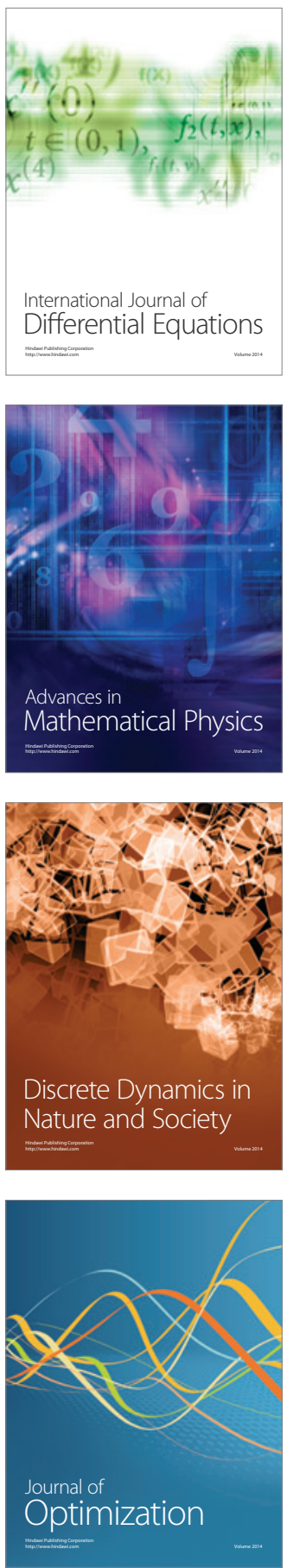